\title{
Structural, Electronic, Stability and Optical Properties of $\mathrm{CsPb}_{1-\mathrm{x}} \mathrm{Sn}_{\mathrm{x}} \mathrm{IBr}_{2}$ Perovskites: A First-Principles Investigation
}

Sinan Zhu, Jinting Ye, Yuanyuan Zhao, and Yongqing Qiu*

Institute of Functional Material Chemistry, Faculty of Chemistry, Northeast Normal University, Changchun, 130024, China

${ }^{*}$ Corresponding Author. Fax: +86 43185098768.

E-mail addresses: qiuyq466@nenu.edu.cn (Y. Q. Qiu) 
Table S1. The atomic coordinatess of $\mathrm{Br}, \mathrm{Cs}$ and $\mathrm{Pb}$ elements in orthorhombic $\mathrm{CsPb}_{1-\mathrm{x}} \mathrm{Sn}_{\mathrm{x}} \mathrm{IBr}_{2}(\mathrm{x}=0,0.25,0.5$ and 0.75$)$ structures.

\begin{tabular}{|c|c|c|c|c|c|c|}
\hline & & & $\mathbf{x}$ & $\mathbf{y}$ & $\mathbf{z}$ & Wyckoff Position \\
\hline 1 & $\mathrm{Br}$ & $\mathrm{Br} 1$ & 0.797 & 0.206 & 0.038 & $8 \mathrm{~d}$ \\
\hline 2 & $\mathrm{Br}$ & $\mathrm{Br} 2$ & 0.297 & 0.294 & 0.962 & $8 \mathrm{~d}$ \\
\hline 3 & $\mathrm{Br}$ & $\mathrm{Br} 3$ & 0.203 & 0.794 & 0.538 & $8 \mathrm{~d}$ \\
\hline 4 & $\mathrm{Br}$ & $\mathrm{Br} 4$ & 0.703 & 0.706 & 0.462 & $8 \mathrm{~d}$ \\
\hline 5 & $\mathrm{Br}$ & $\mathrm{Br} 5$ & 0.203 & 0.794 & 0.962 & $8 \mathrm{~d}$ \\
\hline 6 & $\mathrm{Br}$ & Br6 & 0.703 & 0.706 & 0.038 & $8 \mathrm{~d}$ \\
\hline 7 & $\mathrm{Br}$ & $\mathrm{Br} 7$ & 0.797 & 0.206 & 0.462 & $8 \mathrm{~d}$ \\
\hline 8 & $\mathrm{Br}$ & Br8 & 0.297 & 0.294 & 0.538 & $8 \mathrm{~d}$ \\
\hline 9 & $\mathrm{Br}$ & $\mathrm{Br} 9$ & 0.074 & 0.515 & 0.250 & $8 \mathrm{~d}$ \\
\hline 10 & $\mathrm{Br}$ & $\operatorname{Br} 10$ & 0.574 & 0.986 & 0.750 & $8 \mathrm{~d}$ \\
\hline 11 & $\mathrm{Br}$ & $\operatorname{Br} 11$ & 0.926 & 0.486 & 0.750 & $8 \mathrm{~d}$ \\
\hline 12 & $\mathrm{Br}$ & $\mathrm{Br} 12$ & 0.426 & 0.015 & 0.250 & $8 \mathrm{~d}$ \\
\hline 13 & $\mathrm{Cs}$ & Cs1 & 0.983 & 0.942 & 0.250 & $8 \mathrm{~d}$ \\
\hline 14 & Cs & $\mathrm{Cs} 2$ & 0.483 & 0.558 & 0.750 & $8 \mathrm{~d}$ \\
\hline 15 & Cs & Cs 3 & 0.017 & 0.058 & 0.750 & $8 \mathrm{~d}$ \\
\hline 16 & Cs & $\mathrm{Cs} 4$ & 0.517 & 0.442 & 0.250 & $8 d$ \\
\hline 17 & $\mathrm{~Pb}$ & $\mathrm{~Pb} 1$ & 0.500 & 0.000 & 0.000 & $4 b$ \\
\hline 18 & $\mathrm{~Pb}$ & $\mathrm{~Pb} 2$ & 0.000 & 0.500 & 0.000 & $4 a$ \\
\hline 19 & $\mathrm{~Pb}$ & $\mathrm{~Pb} 3$ & 0.500 & 0.000 & 0.500 & $4 a$ \\
\hline 20 & $\mathrm{~Pb}$ & $\mathrm{~Pb} 4$ & 0.000 & 0.500 & 0.500 & $4 b$ \\
\hline
\end{tabular}


Table S2. The atomic coordinatess of $\mathrm{Br}$, $\mathrm{Cs}$ and $\mathrm{Pb}$ elements in cubic $\mathrm{CsPb}_{1-\mathrm{x}} \mathrm{Sn}_{\mathrm{x}} \mathrm{IBr}_{2}$ $(\mathrm{x}=0,0.25,0.5$ and 0.75$)$ structures.

\begin{tabular}{|c|c|c|c|c|c|c|}
\hline & & & $\mathbf{x}$ & $\mathbf{y}$ & $\mathbf{z}$ & Wyckoff Position \\
\hline 1 & $\mathrm{Br}$ & $\mathrm{Br} 1$ & 0.000 & 0.250 & 0.500 & $12 \mathrm{~h}$ \\
\hline 2 & $\mathrm{Br}$ & $\mathrm{Br} 2$ & 0.250 & 0.000 & 0.500 & $12 \mathrm{~h}$ \\
\hline 3 & $\mathrm{Br}$ & $\mathrm{Br} 3$ & 0.250 & 0.250 & 0.000 & $12 \mathrm{i}$ \\
\hline 4 & $\mathrm{Br}$ & $\mathrm{Br} 4$ & 0.500 & 0.250 & 0.500 & $6 f$ \\
\hline 5 & $\mathrm{Br}$ & $\operatorname{Br} 5$ & 0.750 & 0.000 & 0.500 & $12 \mathrm{~h}$ \\
\hline 6 & $\mathrm{Br}$ & Br6 & 0.750 & 0.250 & 0.000 & $12 \mathrm{i}$ \\
\hline 7 & $\mathrm{Br}$ & $\mathrm{Br} 7$ & 0.000 & 0.750 & 0.500 & $12 \mathrm{~h}$ \\
\hline 8 & $\mathrm{Br}$ & $\mathrm{Br} 8$ & 0.250 & 0.500 & 0.500 & $6 f$ \\
\hline 9 & $\mathrm{Br}$ & $\mathrm{Br} 9$ & 0.250 & 0.750 & 0.000 & $12 \mathrm{i}$ \\
\hline 10 & $\mathrm{Br}$ & $\operatorname{Br} 10$ & 0.500 & 0.750 & 0.500 & $6 f$ \\
\hline 11 & $\mathrm{Br}$ & Br11 & 0.750 & 0.500 & 0.500 & $6 f$ \\
\hline 12 & $\mathrm{Br}$ & $\operatorname{Br} 12$ & 0.750 & 0.750 & 0.000 & $12 \mathrm{i}$ \\
\hline 13 & $\mathrm{Cs}$ & Cs1 & 0.000 & 0.000 & 0.000 & 1a \\
\hline 14 & Cs & Cs2 & 0.500 & 0.000 & 0.000 & $3 d$ \\
\hline 15 & Cs & Cs3 & 0.000 & 0.500 & 0.000 & $3 d$ \\
\hline 16 & Cs & Cs4 & 0.500 & 0.500 & 0.000 & $3 c$ \\
\hline 17 & $\mathrm{~Pb}$ & $\mathrm{~Pb} 1$ & 0.250 & 0.250 & 0.500 & $12 \mathrm{j}$ \\
\hline 18 & $\mathrm{~Pb}$ & $\mathrm{~Pb} 2$ & 0.750 & 0.250 & 0.500 & $12 \mathrm{j}$ \\
\hline 19 & $\mathrm{~Pb}$ & $\mathrm{~Pb} 3$ & 0.250 & 0.750 & 0.500 & $12 j$ \\
\hline 20 & $\mathrm{~Pb}$ & $\mathrm{~Pb} 4$ & 0.750 & 0.750 & 0.500 & $12 \mathrm{j}$ \\
\hline
\end{tabular}


Table S3. Calculated lattice parameters and selected volumes of unit-cell $\left(\AA^{3}\right)$ for two phases mixed $\mathrm{CsPb}_{1-\mathrm{x}} \mathrm{Sn}_{\mathrm{x}} \mathrm{IBr}_{2}(\mathrm{x}=0,0.25,0.5$ and 0.75$)$ structures.

\begin{tabular}{|c|c|c|c|c|c|c|}
\hline & structure & $\mathbf{a}$ & $\mathbf{b}$ & c & $\boldsymbol{V}$ & $E(\mathrm{eV})$ \\
\hline \multirow{5}{*}{$\alpha$-phase } & $\mathrm{CsPbBr}_{3}$ & 11.920 & 11.920 & 6.162 & 875.55 & -63.227 \\
\hline & $\mathrm{CsPbIBr}_{2}$ & 11.921 & 12.750 & 6.161 & 936.37 & -60.690 \\
\hline & $\mathrm{CsPb}_{0.75} \mathrm{Sn}_{0.25} \mathrm{IBr}_{2}$ & 11.864 & 12.693 & 6.145 & 925.35 & -60.581 \\
\hline & $\mathrm{CsPb}_{0.5} \mathrm{Sn}_{0.5} \mathrm{IBr}_{2}$ & 11.807 & 12.635 & 6.126 & 913.94 & -60.477 \\
\hline & $\mathrm{CsPb}_{0.25} \mathrm{Sn}_{0.75} \mathrm{IBr}_{2}$ & 11.747 & 12.578 & 6.108 & 902.43 & -60.390 \\
\hline \multirow{5}{*}{$\gamma$-phase } & $\mathrm{CsPbBr}_{3}$ & 8.518 & 11.882 & 8.240 & 833.90 & -64.218 \\
\hline & $\mathrm{CsPbIBr}_{2}$ & 8.797 & 11.876 & 8.522 & 890.26 & -61.622 \\
\hline & $\mathrm{CsPb}_{0.75} \mathrm{Sn}_{0.25} \mathrm{IBr}_{2}$ & 8.761 & 11.835 & 8.515 & 882.86 & -61.484 \\
\hline & $\mathrm{CsPb}_{0.5} \mathrm{Sn}_{0.5} \mathrm{IBr}_{2}$ & 8.737 & 11.799 & 8.490 & 875.20 & -61.352 \\
\hline & $\mathrm{CsPb}_{0.25} \mathrm{Sn}_{0.75} \mathrm{IBr}_{2}$ & 8.688 & 11.769 & 8.491 & 868.22 & -61.236 \\
\hline
\end{tabular}
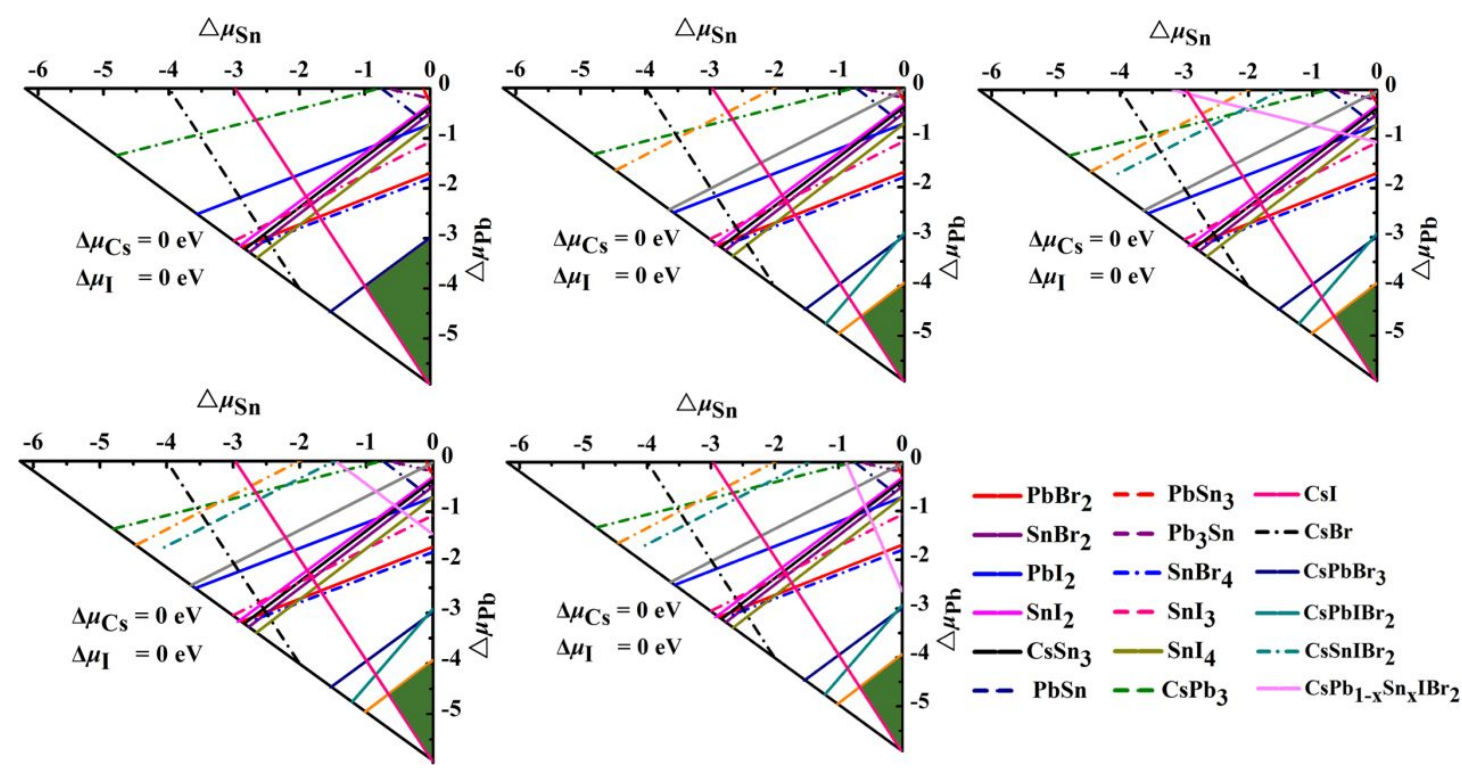

Figure S1. Phase stability diagram analysis results sliced for orthorhombic phase $\mathrm{CsPb}_{1-\mathrm{x}} \mathrm{Sn}_{\mathrm{x}} \mathrm{IBr}_{2}$. The polygon region in green stands for the thermodynamic stable condition and each line are all corresponding to one competing phase. 

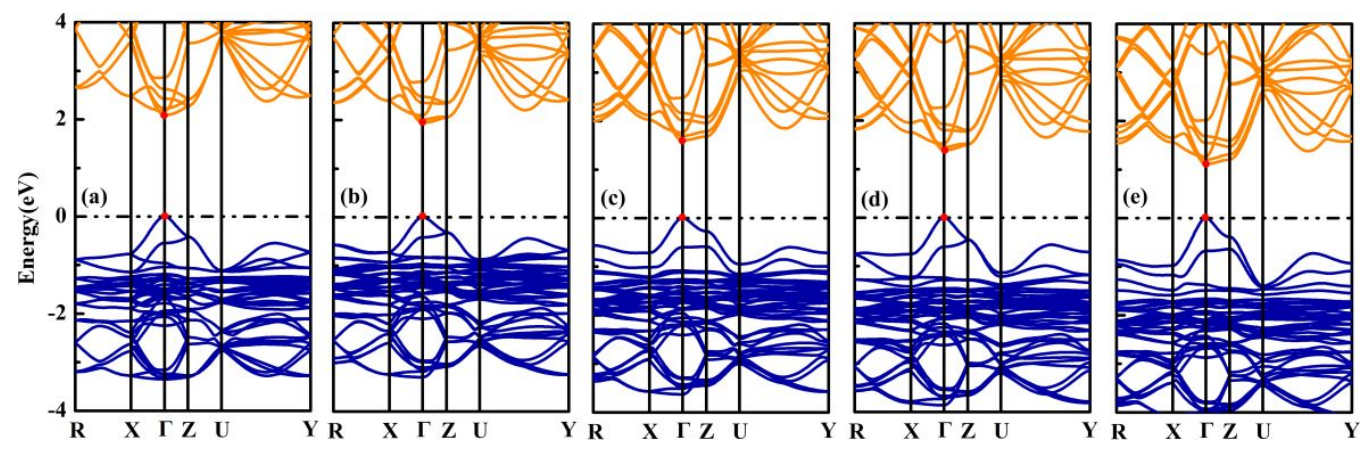

Figure S2. The calculated band structures of orthorhombic phases mixed $\mathrm{CsPb}_{1-\mathrm{x}} \mathrm{Sn}_{\mathrm{x}} \mathrm{IBr}_{2}$ perovskites without SOC.
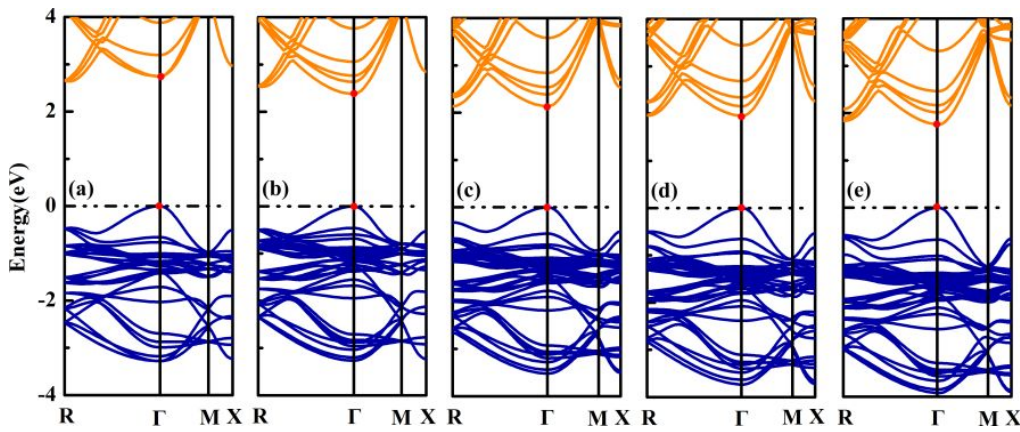

Figure S3. The calculated band structures of cubic phases mixed $\mathrm{CsPb}_{1-\mathrm{x}} \mathrm{Sn}_{\mathrm{x}} \mathrm{IBr}_{2}$ perovskites without SOC.

Table S4. Calculated band gaps with PBE (GGA), HSE06 and PBE (SOC) methods of cubic phase mixed $\mathrm{CsPb}_{1-\mathrm{x}} \mathrm{Sn}_{\mathrm{x}} \mathrm{IBr}_{2}$ perovskites.

\begin{tabular}{|c|c|c|c|c|c|}
\hline & Compound & PBE(GGA) & HSE+GGA & GGA+SOC & Expt \\
\hline \multirow{5}{*}{$\alpha$-phase } & $\mathrm{CsPbBr}_{3}$ & 2.540 & 2.457 & 1.889 & 2.300 \\
\hline & $\mathrm{CsPbIBr}_{2}$ & 2.380 & 2.256 & 1.724 & \\
\hline & $\mathrm{CsPb}_{0.75} \mathrm{Sn}_{0.25} \mathrm{IBr}_{2}$ & 2.136 & 1.835 & 1.626 & \\
\hline & $\mathrm{CsPb}_{0.5} \mathrm{Sn}_{0.5} \mathrm{IBr}_{2}$ & 1.954 & 1.768 & 1.557 & \\
\hline & $\mathrm{CsPb}_{0.25} \mathrm{Sn}_{0.75} \mathrm{IBr}_{2}$ & 1.761 & 1.705 & 1.495 & \\
\hline
\end{tabular}


Table S5. Calculated static dielectric constant $\left(\varepsilon_{\text {std }}\right)$, the electronic $\left(\varepsilon_{\infty}\right)$ and the ionic $\left(\varepsilon_{\text {ion }}\right)$ contributions to the static dielectric constant of mixed $\mathrm{CsPb}_{1-\mathrm{x}} \mathrm{Sn}_{\mathrm{x}} \mathrm{IBr}_{2}$ structures.

\begin{tabular}{cccccccccc}
\hline & \multicolumn{3}{c}{$\varepsilon_{\text {ion }}$} & & & $\varepsilon_{\infty}$ & & & $\varepsilon_{s t d}$ \\
\cline { 2 - 8 } & $\varepsilon_{\text {ion }}^{x x}$ & $\varepsilon_{\text {ion }}^{y y}$ & $\varepsilon_{\text {ion }}^{z z}$ & $\varepsilon_{\infty}^{x x}$ & $\varepsilon_{\infty}^{y y}$ & $\varepsilon_{\infty}^{z z}$ & $\varepsilon_{\text {std }}^{x x}$ & $\varepsilon_{\text {std }}^{y y}$ & $\varepsilon_{\text {std }}^{z z}$ \\
\hline$\alpha-\mathrm{CsPbr}_{3}$ & 20.84 & 20.83 & 1.59 & 4.36 & 4.36 & 3.88 & 25.20 & 25.19 & 5.47 \\
$\alpha-\mathrm{CsPbIBr}_{2}$ & 24.27 & 24.50 & 2.35 & 4.69 & 5.84 & 7.50 & 28.96 & 30.34 & 9.85 \\
$\alpha-\mathrm{CsPb}_{0.75} \mathrm{Sn}_{0.25} \mathrm{IBr}_{2}$ & 47.31 & 24.64 & 3.33 & 5.14 & 6.70 & 8.73 & 52.51 & 31.34 & 12.06 \\
$\alpha-\mathrm{CsPb}_{0.5} \mathrm{Sn}_{0.5} \mathrm{IBr}_{2}$ & 18.53 & 34.51 & 4.12 & 5.86 & 7.89 & 10.4 & 24.39 & 42.40 & 14.52 \\
$\alpha-\mathrm{CsPb}_{0.25} \mathrm{Sn}_{0.75} \mathrm{IBr}_{2}$ & 22.33 & 52.82 & 4.20 & 6.69 & 10.4 & 12.7 & 29.02 & 63.22 & 16.90 \\
\hline$\gamma-\mathrm{Cs} P b B r_{3}$ & 16.13 & 17.13 & 7.91 & 4.01 & 4.08 & 3.91 & 20.14 & 21.21 & 11.82 \\
$\gamma-\mathrm{CsPbIBr}_{2}$ & 10.20 & 8.34 & 25.08 & 4.66 & 4.76 & 4.67 & 14.86 & 13.10 & 29.75 \\
$\gamma-\mathrm{CsPb}_{0.75} \mathrm{Sn}_{0.25} \mathrm{IBr}_{2}$ & 14.99 & 13.16 & 7.67 & 4.98 & 5.06 & 5.02 & 19.97 & 18.22 & 12.69 \\
$\gamma-\mathrm{CsPb}_{0.5} \mathrm{Sn}_{0.5} \mathrm{IBr}_{2}$ & 13.14 & 20.57 & 16.25 & 5.31 & 5.39 & 5.58 & 18.45 & 25.96 & 21.83 \\
$\gamma-\mathrm{CsPb}_{0.25} \mathrm{Sn}_{0.75} \mathrm{IBr}_{2}$ & 17.73 & 21.58 & 9.48 & 5.93 & 5.94 & 6.10 & 23.66 & 27.52 & 15.58 \\
\hline
\end{tabular}
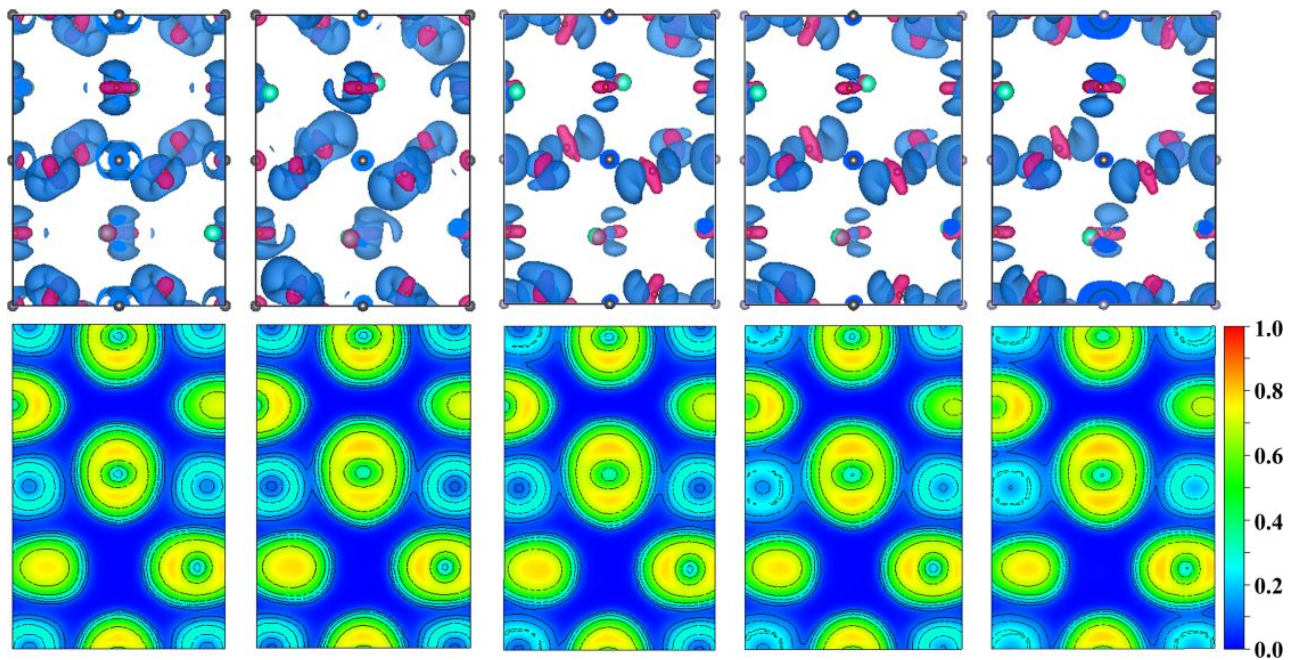

Figure S4. The upper line shows 3D isosurface plots, whereas the blue (red) color represents positive (negative) values and the lower panel shows 2 isoline plots on the middle plane shows the contours of ELF on the (010) plane of orthorhombic phases

$$
\mathrm{CsPb}_{1-\mathrm{x}} \mathrm{Sn}_{\mathrm{x}} \mathrm{IBr}_{2} \text { cells. }
$$

